\title{
Über die Bestimmung des Wismuts als Metall.
}

\section{Von Albin Kurtenacker und Felicitas Werner.}

Die vorliegende Arbeit wurde ausgeführt, um zı untersuchen, ob sich auf die Fällung des Wismuts als Metall eine mabanalytische Methode zur Bestimmung des Wismuts gründen ließe. Eine derartige Nethode müBte viel allgemeiner anwendbar sein, als die bisher empfohlenen Methoden, die auf der Fällung unlöslicher Salze beruhen, denn die Begleitmetalle des Wismuts werden im allgemeinen nicht leicht zu Metall reduziert; sie wirken also nicht störend.

Nach einer Bemerkung von C. MeINEkE ${ }^{1}$ ) hat DE KonINCK bereits versucht, das Wismut durch Zinnchlorür als Metall zu füllen und dann dieses Metall durch Ferrisulfat und Permanganat zu titrieren. Er erhielt keine befriedigenden Resultate.

Wir fanden, daB salzsaure Ferrichloridlösung zur Auflösung des Wismuts viel besser geeignet ist als Ferrisulfat. In letzterer Lösung scheiden sich auf dem zu lösenden Metall basische Wismutsalze $a b$, die die weitere Einwirkung des Ferrisalzes erschweren, so dab die Auflösung nach vielen Stunden noch nicht beendet ist. In der Ferrichloridlösung dagegen löst sich gefälltes Wismutmetall schon bei Zimmertemperatur leicht auf, in der Würme ist die Auflösung in wenigen Minuten beendet. Aber auch Bohrspäne von Handelswismut lösen sich in der Wärme relativ leicht. Wir prüften die Richtigleit der Titration mit reinem, von der Firma KAнLBaUm bezogenen Wismutmetall. Gewogene Proben wurden in der Eisenchloridlösung gelöst und das entstandene Ferrosalz mit Permanganat titriert. Der Permanganatrerbrauch entsprach genau der nach der Gleichung: $\mathrm{Bi}+3 \mathrm{Fe}^{\cdots \cdots}=\mathrm{Bi}^{\cdots}+3 \mathrm{Fe}^{*}$ geforderten Menge.

Nun untersuchten wir die aus Wismutnitratlösungen mit Formaldehyd, unterphosphoriger Säure oder Alkalistannit gefällten Metallniederschläge auf die angegebene Art. Die erhaltenen Resultate waren bis $5 \%$ zu niedrig; außerdem stimmten sie auch unter-

1) De Koninck-Maingre, Lehrbuch der chemischen Analyse, II. Bd., S. 49. 
einander schlecht überein. Wenn man berücksichtigt, daB durch Wägung der mit obigen Reduktionsmitteln gefällten Niederschläge richtige oder um einige Zehntel Prozente zu hohe Werte erhalten werden, so sind die Titrationsergebnisse nur so erklärbar, daB die Niederschläge nicht aus reinem Metall bestehen, sondern daB sie mit Wismutoxydul verunreinigt sind. Ein Gehalt ron Oxydul muB in den Titrationsergebnissen sehr stark zum Ausdruck kommen, da das Wismutoxydul eine dreimal kleinere Menge Ferrisalz reduziert als Wismutmetall. Durch Wägung läBt sich die Anwesenheit geringer Mengen Oxydul im Wismutmetall dagegen schwer feststellen, weil beide ein nur wenig verschiedenes Gewicht haben. Eine Beimengung von 10\% Oxydul erhöht das Gewicht des Niederschlages nur um $0,77 \%$, während dieselbe Menge Oxydul bei der maB. analytischen Bestimmung um $6,67 \%$ zu niedrige Werte bedingt.

L. MOSER ${ }^{1}$ ) hat bereits vermutet, daB der mit Formaldehyd gefallte Niederschlag oxydulhaltig sei und er konnte die Anwesenheit von Oxydul durch das Verhalten des Niederschlages gegenüber Lauge wahrscheinlich machen. Auch die Frage, ob bei der Reduktion mit Stannit, Wismutmetall oder -0xydul entsteht, war Gegenstand vielfacher Untersuchungen, ${ }^{2}$ ) die schlieBlich zu dem Ergebnis führten, daB zumindest bei Anwendung eines Überschusses an Stannit nur Wismutmetall gebildet werden soll.

Die unten anzuführenden Versuche beweisen, daB sich mit jedem der untersuchten Reduktionsmittel oxydulhaltiges Mretall abscheidet und dies auch, wenn man die Reduktionsmittel in groBem ÜberschuB anwendet. Da der Oxydulgehalt bei der maBanalytischen Bestimmung groBe Fehler verursacht, kann die Methode zur Titration des Wismuts nicht angewendet werden.

Wir führten besonders mit Formaldehyd als Fällungsmittel zahlreiche Versuche aus, am durch Änderung der Fällungsbedingungen quantitative Reduktion zu Metall zu erzielen. Die Bemühungen blieben erfolglos. Es gelang nicht einmal unter vollständiger Gleichhaltung der Fällungsbedingungen übereinstimmende Resultate zu erhalten, vielmehr wiesen die Resultate auch hier Differenzen bis zu $3 \%$ auf.

Erst nach Auffindung der Bedingungen für die vollständige

1) L. Moser, Die Bestimmungsmethoden des Wismuts, Seite 41.

2) Literatur hierüber, siehe GunLIN-K $\mathbf{K}_{\text {BUUT, }}$, Handbuch der anorg. Chemie, 7. Auf., Bd. III/2, S. 951. 
Reduktion des Wismuts zu Metall wird es möglich sein, die Metallfällung zur Grundlage einer vielseitig anwendbaren Titrationsmethode für Wismut za machen.

Versuche.

1. Nachstehende Mengen Wismut (KAHLBADM) wurden in Kohlensüure-Atmosphäre in je $20 \mathrm{ccm}$ einer Eisenchloridlösung gelüst, welche $200 \mathrm{~g} \mathrm{FeCl}_{3}$ und $250 \mathrm{ccm}$ konz. Salzsäure pro Liter enthielt. Nach vollendeter Lösung wurde stark verdünnt, mit Mangansulfat-Phosphorsäure versetzt und in einer Porzellanschale mit Permanganat titriert. Die beim Verdünnen ausfallenden basischen Wismutsalze erschwerten das Erkennen des Titrationsendes nicht. Die Eisenchloridlösung selbst verbrauchte kein Permanganat. Einwage: $0,2188 \mathrm{~g} \mathrm{Bi}$; verbraucht $31,59 \mathrm{cem} \mathrm{n} / 10 \mathrm{KMnO}$ entsprech. $0,2191 \mathrm{~g} \mathrm{Bi}$ " $0,2327 ", \quad$ " $" 33,64 \quad$ " " $\quad 0,2333$ ",

"

2. Gewichtsanalytische Bestimmung mit Formaldehyd. Diese Methode wurde ron L. Vanino und F. TreUBent ") empfohlen. Moser (1. c.) und Treadwelu ${ }^{2}$ ) erhielten nach der Methode etwas zu hohe Werte, die Moser, wie oben bemerkt, auf Verunreinigung des Niederschlages mit Wismutoxydul zurückführt, während TREaDWELL dieselben mit Okklusion von Alkali erklärt. Wir erhielten nach der Methode ebenfalls etwas zu hohe Werte:

$19,98 \mathrm{ccm} \mathrm{Bi}\left(\mathrm{NO}_{3}\right)_{3}$-Lösung, entspr. 0,1364 $\mathrm{g} \mathrm{Bi}$ gaben 0,1366 g Bi, d. i. 100,1\% $29,95 ", " \quad " \quad 0,2045 ", " 0,2054 ", " 100,5$,

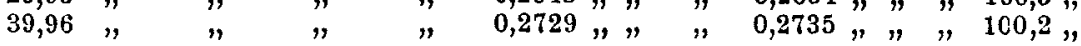

Moser konnte im Filtrat vom Wismutniederschlag mit $\mathrm{S}^{\prime \prime}$-Ionen stets noch Wismut nachweisen. Wir schieden das Wismut aus dem Filtrat als Sultid $a b$ und bestimmten es einigemale. Wir fanden, daB in ungünstigen Füllen etwa $1 \%$ der angewandten Wismutmenge ins Filtrat übergeht, daB sich diese Menge aber durch Anwendung genügender Formaldehydkonzentration bei der Fällung auf unwägbare Spuren herabdrücken läBt.

3. MaBanalytische Bestimmung mit Formaldehyd als Reduktionsmittel. Schwachsaure Wismutnitratlösung wurde mit Wasser auf $100 \mathrm{ccm}$ verdünnt und mit Lauge annähernd neatralisiert. Hierauf wurden 5-10 ccm Formaldehyd $(40 \%)$ und $20-40 \mathrm{ccm}$ Natron-

1) Berl. Ber. 31 (1898), 1903.

8) Treadweld, Lehrbuch der analytiachen Chemie, II. Bd., 9. Aufl, S. 151. 
lauge $(10 \%)$ zugegeben. Nun wurde anf dem Wasserbade so lange erhitzt, bis die über dem Niederschlage befindliche Flüssigkeit vollkommen klar geworden war. Bei längerem Erhitzen, besonders bei Anwendung hoher Formaldehyd- und Laugenkonzentration färbt sich die Flüssigkeit gelb bis tief brannrot, ohne daB damit eine merkliche Anderung der Resultate verbunden wäre. Der mit einem Glasstab zu einem Metallklumpen vereinigte Niederschlag wurde durch Dekantation mit heiBem Wasser ausgewaschen, in Eisenchloridlösung gelöst und wie oben titriert.

Bei Anwendung von $20-40 \mathrm{ccm}$ Wismutlösung, entsprechend 0,1365-0,2730 $\mathrm{g}$ Wismut, erhielten wir, wenn die Fällung mit $5 \mathrm{ccm}$ Formaldehyd und $20 \mathrm{ccm}$ Lauge bewirkt wurde, im Mittel von 23 Versuchen $96,4 \%$ der angewandten Wismutmenge. $8 \mathrm{Be}$ stimmungen lagen zwischen 95,1 und $96,0 \%, 8$ Bestimmungen zwischen 96,1 und $97,0 \%, 7$ Bestimmungen zwischen 97,1 und $97,6 \%$.

Wurde mit $10 \mathrm{ccm}$ Formaldehyd und $20 \mathrm{~cm}$ Lauge gefällt, so ergaben 10 Versuche im Mittel $97,3 \%$ statt 100 . Davon lagen 2 Werte bei 95,2 resp. $96,3 \%, 6$ Werte zwischen 96,9 und $97,7 \%$ und 2 Werte bei $98,5 \%$.

Höhere Lauge- und Formaldehydkonzentration hatte auf die Resultate keinen merkbaren EinfluB, ebenso worden die Resultate durch die Art der Fällnng, die Verdünnung der Wismutlösung, die Dauer des Erhitzens u. dgl. nicht beeinflußt.

4. Reduktion mit unterphosphoriger Säure. Dieses Reduktionsmittel wurde von W. Muthmañ und F. MaWROW ${ }^{1}$ ) für die gewichtsanalytische Bestimmung des Wismuts vorgeschlagen. Wir versetzten eine abgemessene Zahl Kubikzentimeter Wismutnitratlösung mit $10 \mathrm{ccm}$ Natriumhypophosphitlösung $(50 \%)$ und erhitzten auf dem Wasserbade bis zum Klarwerden der über dem Niederschlage befindlichen Flüssigkeit. Dann wurde filtriert und weiter wie unter 3 angegeben, rerfahren:

$$
\mathrm{Bi}\left(\mathrm{NO}_{3}\right)_{3} \mathrm{Bi} \quad \mathrm{n} / 10 \mathrm{KMnO}, \quad \mathrm{Bi}
$$

$20,05 \mathrm{ccm}$, entspr. $0,1369 \mathrm{~g}$, verbr, $19,25 \mathrm{ccm}$, entspr. $0,1335 \mathrm{~g}$, d. i. $97,5 \%$

$30 "$ " $" 0,2047 "$ " $28,68,0,1989, " 97,1$ "

$40 " \quad " \quad 0,2731 " \quad " 38,98, \quad " \quad 0,2704, \quad$ " $" 99,0$ "

Mit Ausnahme des letzten Versuches gaben die Filtrate mit Natriumsulfid Wismutreaktion.

1) Z. anorg. Chem. 13 (1897), 209.

Z. snorg. n. allg. Chem. Bd. 123. 
170 A. Kurtenacker und F. Werner. Bestimmung des Wismuts als Metall.

5. Reduktion mit Stannit. Die Wismutlösung wurde mit $10 \mathrm{ccm}$ Weinsäurelösung $(10 \%)$ und $20 \mathrm{ccm}$ Natronlauge $(10 \%)$ versetzt. $\mathrm{Zu}$ der klaren Lösung wurde die doppelte Zahl Kubikzentimeter einer Stannitlösung zugegeben, die hergestellt wurde durch Auflösen von $9 \mathrm{~g} \mathrm{SnCl}_{2} \cdot 2 \mathrm{H}_{2} \mathrm{O}$ und $30 \mathrm{~g}$ Weinsäure in Lauge und Auffüllen auf $400 \mathrm{ccm}$. Nun wurde auf dem Wasserbade erhitzt und weiter wie oben verfahren:

\begin{tabular}{|c|c|c|c|c|c|c|c|c|}
\hline $\mathrm{Bi}\left(\mathrm{NO}_{3}\right)_{3}$ & & $\mathrm{Bi}$ & & $\mathrm{n} / 10 \mathrm{KMnO}$ & & $\mathrm{Bi}$ & & \\
\hline $\begin{array}{l}20,05 \mathrm{ccm}, \\
40,08 \quad " \\
20,10 "\end{array}$ & $\begin{array}{c}\text { entspt. } \\
\text { " }\end{array}$ & $\begin{array}{l}0,1369 \mathrm{~g}, \\
0,2736 ", \\
0,1372\end{array}$ & verbr. & $\begin{array}{l}19,49 \mathrm{ccm}, \\
38,50 \quad " \\
19,35 \quad "\end{array}$ & $\begin{array}{l}\text { entspr. } \\
" "\end{array}$ & $\begin{array}{l}0,1352 \mathrm{~g}, \\
0,2669 " \\
0,1342\end{array}$ & d. i. & $\begin{array}{l}98,8 \% \\
\mathbf{9 7 , 5} \% \\
\mathbf{9 7 , 8} \%\end{array}$ \\
\hline
\end{tabular}

Brünn, Deutsche technische Hochschule, Laboratorium für anorganische, physikalische und analytische Chemie.

Bei der Redaktion eingegangen am 30. Mai 1922. 